\title{
GLUT1 as a Prognostic Factor for Classical Hodgkin's Lymphoma: Correlation with PD-L1 and PD-L2 Expression
}

Young Wha Koh · Jae-Ho Han Seong Yong Park ${ }^{1} \cdot$ Dok Hyun Yoon ${ }^{2}$ Cheolwon Suh ${ }^{2} \cdot$ Jooryung $\mathrm{Huh}^{3}$

Departments of Pathology and ${ }^{1}$ Thoracic and Cardiovascular Surgery, Ajou University School of Medicine, Suwon; Departments of ${ }^{2}$ Oncology and ${ }^{3}$ Pathology, Asan Medical Center, University of Ulsan College of Medicine, Seoul, Korea

Received: August 23, 2016

Revised: October 17, 2016

Accepted: November 3, 2016

\section{Corresponding Author}

Jooryung Huh, MD

Department of Pathology, Asan Medical Center,

University of Ulsan College of Medicine,

88 Olympic-ro 43-gil, Songpa-gu, Seoul 05505,

Korea

Tel: +82-2-3010-4553

Fax: +82-2-472-7898

E-mail: jrhuh@amc.seoul.kr

\begin{abstract}
Background: Glucose transporter type 1 (GLUT1) expression is linked to glucose metabolism and tissue hypoxia. A recent study reported that GLUT1 was significantly associated with programmed death ligand 1 (PD-L1) as a therapeutic target in relapsed or refractory classical Hodgkin's lymphoma (cHL). The purpose of this study was to measure the expression of GLUT1 and assess its prognostic significance and potential relationships with PD-L1, programmed death ligand 2 (PD-L2), and programmed death-1 (PD-1) expressions in cHL. Methods: Diagnostic tissues from 125 patients with $\mathrm{CHL}$ treated with doxorubicin, bleomycin, vinblastine, and dacarbazine were evaluated retrospectively via immunohistochemical analysis of GLUT1, PD-L1, PD-L2, and PD-1 expression. Results: The median follow-up time was 4.83 years (range, 0.08 to 17.33 years). GLUT1, PD-L1, PD-L2, and PD-1 were expressed in $44.8 \%, 63.2 \%, 9.6 \%$, and $13.6 \%$ of the specimens, respectively. Positive correlations were found between GLUT1 and PD-L1 expression ( $p=.004)$ and between GLUT1 and PD-L2 expression ( $p=.031$ ). GLUT1 expression in Hodgkin/Reed-Sternberg (HRS) cells was not associated with overall survival or event-free survival (EFS) in the entire cohort $(p=.299$ and $p=.143$, respectively). A subgroup analysis according to the Ann Arbor stage illustrated that GLUT1 expression in HRS cells was associated with better EFS in advanced-stage disease ( $p=$ .029). A multivariate analysis identified GLUT1 as a marginally significant prognostic factor for EFS $(p=.068)$. Conclusions: This study suggests that GLUT1 expression is associated with better clinical outcomes in advanced-stage $\mathrm{CHL}$ and is significantly associated with PD-L1 and PD-L2 expressions.
\end{abstract}

Key Words: Hodgkin lymphoma; GLUT1; Programmed death ligand 1; Programmed death ligand 2; Programmed death-1
Classical Hodgkin lymphoma (cHL) is considered a highly curable disease with modern therapy; however, at least $20 \%$ of the patients cannot be cured with the standard treatment. ${ }^{1,2} \mathrm{Al}-$ though the International Prognostic Score (IPS) is a powerful tool for risk stratification, it does not fully reflect the biological nature of cHL. Furthermore, the prognostic significance of IPS is limited in advanced-stage disease. ${ }^{3}$

Glucose transporter type 1 (GLUT1) is responsible for the cellular glucose uptake, and it is often overexpressed in malignant tumors, which are characterized by an increased glycolytic rate to meet the intense energy requirements for cell proliferation. ${ }^{4}$ GLUT1 overexpression has been correlated with a poor prognosis in several cancers including the lung, ${ }^{5}$ breast, ${ }^{6}$ colon, ${ }^{7}$ and stomach cancers. $^{8}$ Although GLUT1 expression was evaluated in $\mathrm{CHL},{ }^{9,10}$ it was not associated with clinical outcomes in a previous study. Some cancer prediction systems such as the IPS are useful only for certain stages; however, previous studies did not perform subgroup analyses according to the Ann Arbor stage.

The programmed death-1 (PD-1) pathway has emerged as an important mechanism for tumor evasion from anti-tumor immune responses. ${ }^{11}$ Two ligands for PD-1, programmed death ligand 1 (PD-L1) and programmed death ligand 2 (PD-L2), act as negative immune regulators via interactions with the PD-1 receptor. ${ }^{12}$ PD-1/PD-L1 and PD-L2 have been reported as prognostic biomarkers for hepatocellular carcinoma, ${ }^{13}$ urothelial carcinoma, ${ }^{14}$ breast cancer, ${ }^{15}$ lung cancer, ${ }^{16}$ and colorectal cancer. ${ }^{17}$

Hypoxia-inducible factors (HIFs) play an important role in tumor immune responses. ${ }^{18}$ HIF-1a and HIF-2 $\alpha$ stabilized by hypoxia promote the expression of PD-L1 via binding to a specific hypoxia-response element in the promoter of PD-L1 in myeloidderived suppressor cells, dendritic cells, and macrophages, as well as in prostate, breast, and colorectal cancer cell lines. ${ }^{19-22}$ It will be interesting to evaluate the relationship between GLUT1 and PD-L1 because GLUT1 is a well-known target gene of the 
HIF pathways. ${ }^{19,22,23}$ A recent study reported that PD-L1 expression is regulated by GLUT1 in clear cell renal cell carcinoma. ${ }^{24}$ An anti-PD-1 monoclonal antibody (nivolumab) has exhibited substantial therapeutic activity and an acceptable safety profile in relapsed or refractory cHL. ${ }^{25}$ A GLUT1-specific inhibitor (STF-31) also suppressed glucose uptake and induced apoptosis in myeloma cells with high GLUT1 expression. ${ }^{26}$ However, no study has examined the relationship between PD-L1 and GLUT1 in patients with cHL. This retrospective study evaluated the expression of GLUT1 and assessed its prognostic significance and potential relationships with PD-L1, PD-L2, and PD-1 expressions in $\mathrm{CHL}$.

\section{MATERIALS AND METHODS}

\section{Patients}

A retrospective analysis of 125 consecutive patients diagnosed with cHL at Asan Medical Center between 1995 and 2012 and at Ajou University Hospital between 2008 and 2015 was performed. All patients were pathologically confirmed to have $\mathrm{CHL}$ and treated with the doxorubicin, bleomycin, vinblastine, and dacarbazine (ABVD) therapy regimen, with or without radiation. The patients were $\geq 15$ years of age at diagnosis, and they had no previous treatment or history of malignancy. Paraffinembedded tumor tissues and follow-up data were available for all patients included in the study. The median follow-up time was 4.83 years (range, 0.08 to 17.33 years). Response criteria were based on standard guidelines. Routine follow-up imaging analyses were performed every 3 months for the first 2 years, every 6 months for the next 3 years, and annually (or whenever clinically indicated) thereafter. The results of PD-L1, PD-L2, and PD-1 expressions from a previously reported study were used. ${ }^{27}$ The research was approved by the Institutional Review Board of Asan Medical Center.

\section{Histopathological analysis and immunohistochemistry}

All histological and immunophenotypic data were reviewed by two pathologists (J.H. and Y.W.K.). The cases of cHL were subtyped according to the World Health Organization criteria as nodular sclerosis, lymphocyte-rich, mixed cellularity, lymphocyte-depleted, or not otherwise specified cHL. Tissue microarrays (TMAs) were constructed using three 1-mm-diameter tumor cores from selected areas of formalin-fixed, paraffin-embedded tumor samples. TMA sections were stained using a Benchmark XT automatic immunohistochemistry staining device (Ventana Medical Systems, Tucson, AZ, USA). Anti-
GLUT1 (Cell Marque, Rocklin, CA, USA), anti-PD-L1 (Cell Signaling Technology, Danvers, MA, USA), anti-PD-L2 (R\&D Systems, Minneapolis, MN, USA), and anti-PD-1 antibodies (Cell Marque) were used. In the TMA, each case was represented by three tissue cores, and at least 10 CD30-positive Hodgkin/ Reed-Sternberg (HRS) cells in at least one of the three core cylinders from each patient were read. To minimize the counting of cells, other than small lymphocytes, with nonspecific PD-1 staining we counted only the cells with staining that were morphologically compatible with small lymphocytes in the microenvironment (excluded fibroblasts, endothelial cells, HRS cells, and macrophages) based on their size, shape, and CD30 staining. We examined the protein expression of GLUT1, PD-L1, PDL2, and PD-1 in 5\% increments. The cut-off values of GLUT1, PD-L1, PD-L2, and PD-1 associated with the most significant differences in overall survival (OS) were selected. A sample was considered GLUT1-, PD-L1-, or PD-L2-positive if the expression of these markers was detected in $\geq 20 \%$ of HRS cells. A sample was considered PD-1-positive if PD-1 expression was detected in $\geq 20 \%$ of the peritumoral microenvironment. In situ hybridization analysis of Epstein-Barr virus-encoded RNA-1 and RNA-2 was performed and scored as previously described. ${ }^{28}$

\section{Statistical analyses}

Event-free survival (EFS) was defined as the interval between the date of diagnosis and that of disease progression, relapse, or death from any cause. OS was defined as the interval between the date of diagnosis and that of death from any cause. The followup of patients was censored at their last follow-up date. Cumulative OS or EFS was analyzed using the Kaplan-Meier method, and comparisons were performed via log-rank testing. Multivariate prognostic analyses was performed with the Cox proportional hazards regression model using the enter method. Categorical variables were compared using the chi-squared test. All statistical analyses were performed using the SPSS statistical software program ver. 18.0 (SPSS Inc., Chicago, IL, USA). All p-values were two-sided associations, and $\mathrm{p}<.05$ was considered statistically significant.

\section{RESULTS}

\section{Patient characteristics}

The clinical characteristics of the 125 patients are summarized in Table 1. The median age of the patients was 39 years (range, 15 to 78 years). Twenty-five patients died. The median OS was not achieved, and the median EFS was 11 years. The estimated 
Table 1. Demographic and clinical characteristics of patients

\begin{tabular}{lc}
\hline Characteristics at diagnosis & No. of patients (\%) \\
\hline Age, median (range, yr) & $39(15-78)$ \\
Male gender & $74(59.2)$ \\
Histologic subtype & \\
Nodular sclerosis & $83(66.4)$ \\
Mixed cellularity & $27(21.6)$ \\
Lymphocyte-rich & $6(4.8)$ \\
Lymphocyte-depleted & $3(2.4)$ \\
Not classifiable & $6(4.8)$ \\
Ann Arbor stage & \\
I & $26(20.8)$ \\
II & $41(32.8)$ \\
III & $29(23.2)$ \\
IV & $29(23.2)$ \\
Stage (limited vs advanced) & \\
Limited & $48(38.4)$ \\
Advanced & $77(61.6)$ \\
B symptoms present & $41(32.8)$ \\
International Prognostic Score $\geq 3$ (high-risk) & $49(39.2)$ \\
EBER positivity & $49(39.2)$ \\
Primary treatment & \\
Chemotherapy & $95(76.0)$ \\
Chemoradiotherapy & $30(24.0)$ \\
\hline
\end{tabular}

EBER, Epstein-Barr virus-encoded RNA-1 and RNA-2 assessed by in situ hybridization.

5-year OS and EFS rates were $84 \%$ and $61 \%$, respectively.

\section{GLUT1 expression in CHL tissues}

The correlations between GLUT1 and clinical variables are summarized in Table 2. Fifty-six patients (44.8\%) displayed membranous positivity for GLUT1 (Fig. 1A). There was no correlation between GLUT1 expression and clinical variables.

\section{Correlations of PD-L1, PD-L2, and PD-1 expression with GLUT1 expression}

PD-L1, PD-L2, and PD-1 were expressed in 63.2\%, 9.6\%, and $13.6 \%$ of the specimens, respectively (Fig. 1B-D). The correlations of PD-L1, PD-L2, and PD-1 expression with GLUT1 expression are summarized in Table 3. GLUT1 protein expression was associated with high PD-L1 ( $\mathrm{p}=.004)$ and high PD-L2 protein expression in HRS cells $(\mathrm{p}=.031)$. However, there was no correlation between GLUT1 levels in HRS cells and PD-1 expression in the peritumoral microenvironment $(\mathrm{p}=.198)$.

\section{Prognostic significance of GLUT1 expression}

GLUT1 expression was not associated with EFS and OS rates $(\mathrm{p}=.143$ and $\mathrm{p}=.299$, respectively). As the prognostic significance of the IPS is limited in advanced-stage disease, ${ }^{3}$ we performed a
Table 2. Correlations between clinical variables and GLUT1 expression in all cases

\begin{tabular}{|c|c|c|c|}
\hline \multirow[b]{2}{*}{ Characteristic } & \multicolumn{2}{|c|}{ GLUT1 expression } & \multirow[b]{2}{*}{$p$-value } \\
\hline & $\begin{array}{c}\text { Low }(<20 \%) \\
\quad(n=69)\end{array}$ & $\begin{array}{c}\text { High }(\geq 20 \%) \\
\quad(n=56)\end{array}$ & \\
\hline Age (yr) & & & $.858^{\mathrm{a}}$ \\
\hline$<45$ & $38(55.1)$ & $32(57.1)$ & \\
\hline$\geq 45$ & $31(44.9)$ & $24(42.9)$ & \\
\hline Gender & & & $.276^{\mathrm{a}}$ \\
\hline Male & $44(63.8)$ & $30(53.6)$ & \\
\hline Female & $25(36.2)$ & $26(46.4)$ & \\
\hline Disease subtype & & & $.182^{b}$ \\
\hline Nodular sclerosis & $43(62.3)$ & $40(71.4)$ & \\
\hline Mixed cellularity & $14(20.3)$ & $13(23.2)$ & \\
\hline Lymphocyte-rich & $6(8.7)$ & 0 & \\
\hline Lymphocyte-depleted & $2(2.9)$ & $1(1.8)$ & \\
\hline Not classifiable & $4(5.8)$ & $2(3.6)$ & \\
\hline B symptom & & & $.184^{\mathrm{a}}$ \\
\hline Absent & $50(72.5)$ & $34(60.7)$ & \\
\hline Present & $19(27.5)$ & $22(39.3)$ & \\
\hline Ann Arbor stage & & & $.586^{\mathrm{a}}$ \\
\hline Limited & $28(40.6)$ & $20(35.7)$ & \\
\hline Advanced & $41(59.4)$ & $36(64.3)$ & \\
\hline IPS & & & $.467^{\mathrm{a}}$ \\
\hline$<3$ & $44(63.8)$ & $32(57.1)$ & \\
\hline$\geq 3$ & $25(36.2)$ & $24(42.9)$ & \\
\hline EBER & & & $.467^{\mathrm{a}}$ \\
\hline Negative & $44(63.8)$ & $32(57.1)$ & \\
\hline Positive & $25(36.2)$ & $24(42.9)$ & \\
\hline Primary treatment & & & $.674^{\mathrm{a}}$ \\
\hline Chemotherapy & $51(73.9)$ & $44(78.6)$ & \\
\hline Chemoradiotherapy & $18(26.1)$ & $12(21.4)$ & \\
\hline
\end{tabular}

Values are presented as number (\%).

GLUT1, glucose transporter 1; IPS, International Prognostic Score; EBER, Epstein-Barr virus-encoded RNA-1 and RNA-2 assessed by in situ hybridization.

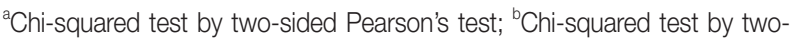
sided Fisher test.

subgroup analysis according to the disease stage to determine whether GLUT1 expression has prognostic significance in certain stages. In limited-stage cHL, GLUT1 expression was not associated with EFS and OS rates ( $\mathrm{p}=.906$ and $\mathrm{p}=.833$, respectively). In advanced-stage cHL, GLUT1 positivity was associated with better EFS ( $\mathrm{p}=.029$ ) (Fig. 2A). However, there was no association between GLUT1 expression and OS in advanced-stage cHL $(\mathrm{p}=.168)($ Fig. 2B).

Univariate analysis revealed that EFS was associated with advanced age and anemia (Table 4). Multivariate analysis identified GLUT1 as a marginally significant prognostic factor for EFS, together with advanced age and anemia (hazard ratio, $0.462 ; \mathrm{p}=$ .068) (Table 4). 

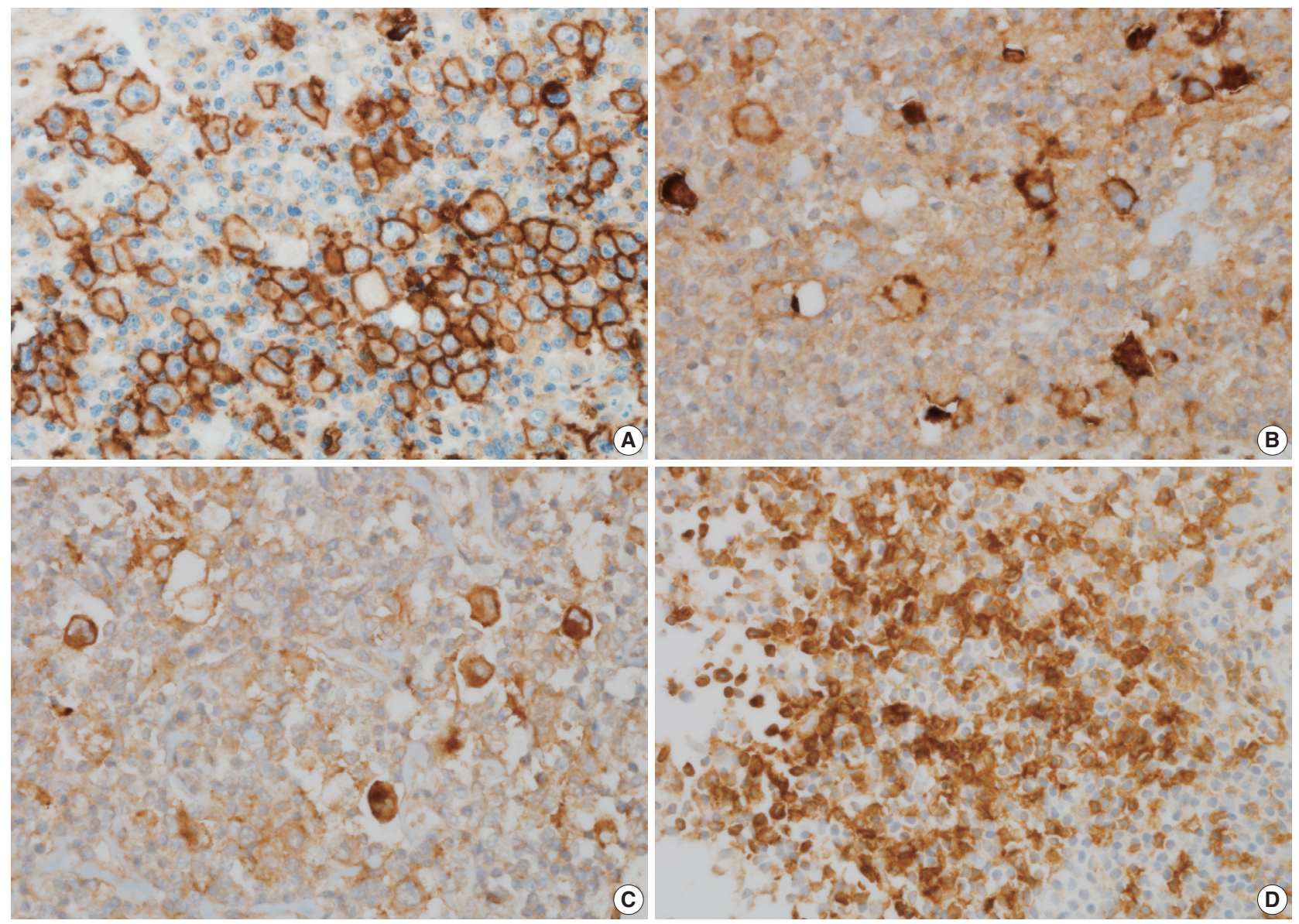

Fig. 1. Glucose transporter type 1 (GLUT1), programmed death ligand 1 (PD-L1), programmed death ligand 2 (PD-L2), and programmed death-1 (PD-1) expression in classical Hodgkin's lymphoma tissues. (A) High GLUT1 expression in Hodgkin/Reed-Sternberg (HRS) cells ( $\geq$ 20\%). (B) High PD-L1 expression in HRS cells ( $\geq 20 \%)$. (C) High PD-L2 expression in HRS cells ( $\geq 20 \%)$. (D) High PD-1 expression in the peritumoral microenvironment $(\geq 20 \%)$.

Table 3. Correlations between GLUT1, PD-L1, PD-L2, and PD-1 expression in all cases

\begin{tabular}{|c|c|c|c|}
\hline \multirow[b]{2}{*}{ Characteristic } & \multicolumn{2}{|c|}{ GLUT1 expression } & \multirow[b]{2}{*}{$p$-value } \\
\hline & $\begin{array}{l}\text { Low }(<20 \%) \\
\quad(n=69)\end{array}$ & $\begin{array}{l}\operatorname{High}(\geq 20 \%) \\
\quad(n=56)\end{array}$ & \\
\hline PD-L1 expression in HRS cells & & & $.004^{a}$ \\
\hline Low (<20\%) & 19 (33.3) & $4(8.9)$ & \\
\hline High ( $\geq 20 \%)$ & $38(66.7)$ & $41(91.1)$ & \\
\hline PD-L2 expression in HRS cells & & & $.299^{\mathrm{a}}$ \\
\hline Low $(<20 \%)$ & $54(94.7)$ & $36(80.0)$ & \\
\hline High ( $\geq 20 \%)$ & $3(5.3)$ & $9(20.0)$ & \\
\hline $\begin{array}{l}\text { PD-1 expression in peritumoral } \\
\text { microenvironment }\end{array}$ & & & $.198^{\mathrm{a}}$ \\
\hline Low $(<20 \%)$ & 57 (82.6) & $51(91.1)$ & \\
\hline High ( $\geq 20 \%)$ & $12(17.4)$ & $5(8.9)$ & \\
\hline
\end{tabular}

Values are presented as number (\%).

GLUT1, glucose transporter type 1; PD-L1, programmed death ligand 1; PD-L2, programmed death ligand 2; PD-1, programmed death-1; HRS, Hodgkin/Reed-Sternberg.

${ }^{a}$ Chi-squared test by two-sided Pearson's test.

\section{DISCUSSION}

In the present study, the expression of GLUT1 was significantly correlated with PD-L1 and PD-L2 expression, supporting the hypothesis that GLUT1-related signaling pathways play an important role in the PD-L1 or PD-L2 pathway. In particular, GLUT1 expression was marginally associated with better EFS in advanced-stage cHL.

There are several explanations for the relationships between PD-1 pathways and GLUT1 expression. HIF-2 $\alpha$ overexpression stabilized by hypoxia increased PD-L1 mRNA and protein levels in a kidney clear cell carcinoma cell line. ${ }^{19} \mathrm{~A}$ previous study also revealed the direct binding of HIF-2 $\alpha$ to a transcriptionally active hypoxia-response element in the human PD-L1 proximal promoter. ${ }^{19}$ GLUT1 is an important downstream target of HIF$2 \alpha .^{19,22}$ Therefore, a significant correlation between GLUT1 and PD-L1 expression was identified in clear cell renal cell carcino- 

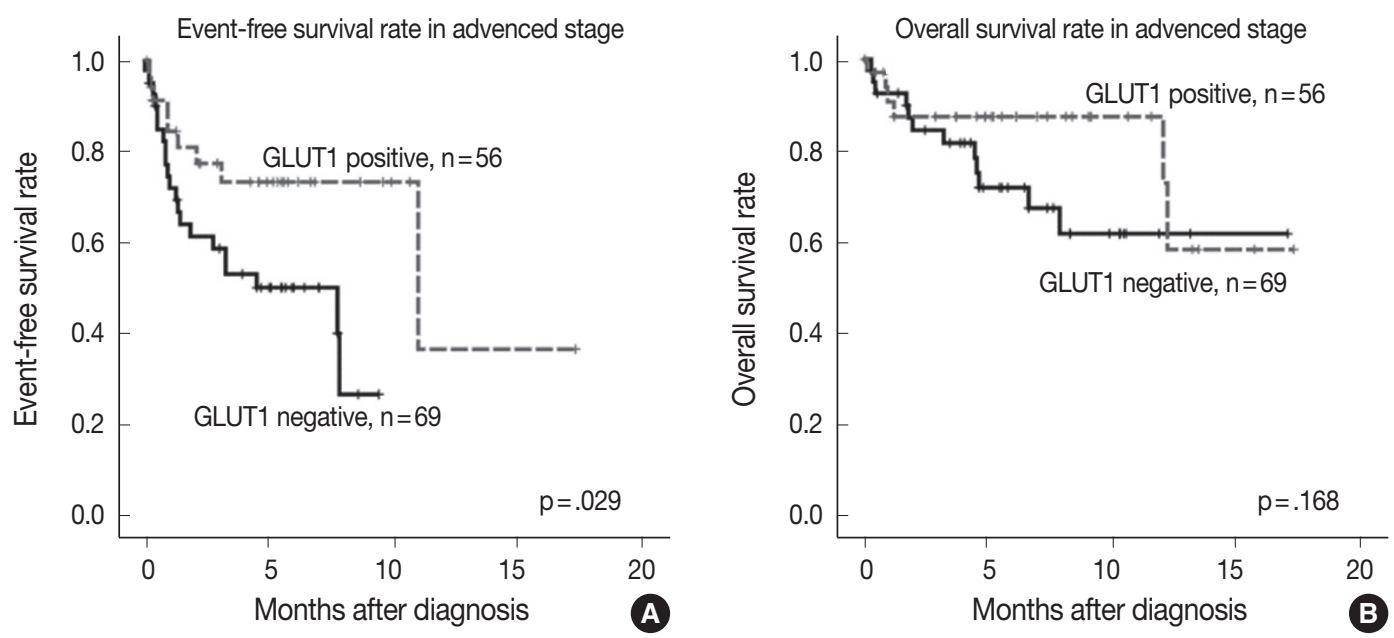

Fig. 2. Comparison of survival rates according to glucose transporter type 1 (GLUT1) expression in advanced-stage classical Hodgkin's lymphoma. The event-free survival rate is significantly higher in the GLUT1-positive group than in the GLUT1-negative group (A). GLUT1 expression is not significantly associated with the overall survival rate $(\mathrm{B})$.

Table 4. Univariate and multivariate analysis of event-free survival in advanced stage

\begin{tabular}{lllll}
\hline Covariate & & HR & $95 \% \mathrm{Cl}$ & $\mathrm{p}$-value \\
\hline Univariate analysis & & & & \\
Age & $<45$ yr vs $\geq 45$ yr & 3.22 & $1.47-7.05$ & .003 \\
Sex & Female vs male & 1.52 & $0.72-3.20$ & .271 \\
B symptoms & $(-)$ vs (+) & 1.85 & $0.86-3.96$ & .112 \\
EBER & $(-)$ vs (+) & 1.64 & $0.94-2.85$ & .081 \\
Lymphopenia & $(-)$ vs (+) & 1.32 & $0.31-5.55$ & .706 \\
Leukocytosis & $(-)$ vs (+) & 1.38 & $0.46-4.14$ & .563 \\
Hypoalbuminemia & $(-)$ vs (+) & 1.65 & .311 \\
Anemia & $(-)$ vs (+) & 2.31 & $0.62-4.31$ & .032 \\
GLUT1 expression & $(-)$ vs (+) & 0.42 & $.07-4.94$ & .035 \\
Multivariate analysis & & & $0.18-0.94$ & .031 \\
Age & $<45$ yr vs $\geq 45$ yr & 2.47 & $1.08-5.61$ & .107 \\
Anemia & $(-)$ vs (+) & 1.92 & $0.86-4.24$ & .068 \\
GLUT1 expression & $(-)$ vs (+) & 0.46 & $0.20-1.05$ & \\
\hline
\end{tabular}

$\mathrm{HR}$, hazard ratio; Cl, confidence interval; EBER, Epstein-Barr virus-encoded RNA-1 and RNA-2 assessed by in situ hybridization; GLUT1, glucose transporter 1 . ${ }^{a}$ Cox univariate analysis.

ma. ${ }^{24}$ In our study, $91 \%$ of patients with GLUT1-positive status had PD-L1 positive status, supporting the HIF-2 $\alpha-$ Glut1-PDL1 pathway identified in a previous study. However, $48 \%$ of patients with PD-L1-positive status had GLUT1 negative status. Further studies are needed to confirm the precise mechanism of the PD-L1 pathway, regardless of GLUT1 expression, in patients with cHL.

In the present study, GLUT1 expression was associated with better EFS in advanced-stage cHL, although the result was of borderline statistical significance. GLUT1 expression was not associated with EFS or OS rates in the entire patient cohort. Hartmann et al. ${ }^{9}$ reported no prognostic significance for GLUT1 expression in cHL. However, Hartmann et al. ${ }^{9}$ did not perform subgroup analysis according to the Ann Arbor stage. Some prognostic biomarkers have limited importance in certain clinical stages. The IPS also has prognostic significance in advanced-stage cHL. Moreover, further research is needed to confirm the prognostic impact of GLUT1 expression.

Although our results revealed a favorable clinical outcome in patients with GLUT1-positive advanced-stage cHL, previous studies revealed correlations between high GLUT1 expression and poor survival in other solid malignancies. ${ }^{5-8}$ In the present study, all patients received the ABVD regimen. Doxorubicin and vinblastine are associated with DNA damage. Doxorubicin inhibits the progression of the enzyme topoisomerase II, which relaxes supercoils in DNA for transcription. ${ }^{29}$ Vinblastine can suppress microtubule dynamics, resulting in mitotic block and apoptosis. ${ }^{30}$ Therefore, HRS cells with higher proliferation rates 
would be more susceptible to chemotherapy-associated DNA damage. GLUT1 overexpression increases glucose metabolism in tumors to enhance cellular proliferation in melanoma and gastric cancer. ${ }^{31,32}$ Some studies ascribed the superior survival of patients with diffuse large B-cell lymphoma or $\mathrm{cHL}$ to increased sensitivity to chemotherapy due to higher cell proliferation rates. ${ }^{33,34}$ Although we did not examine the proliferation rates of HRS cells in our series, the superior survival of cHL patients with GLUT1 expression in our study may imply higher proliferation rates of HRS cells.

Differential expression of GLUT1 was detected according to the histologic type of cHL in a previous study. ${ }^{9}$ GLUT1 expression was more rarely observed in HRS cells in the lymphocyterich classical cHL subtype (30\%) than in the nodular sclerosis (56\%), mixed cellularity (41\%), or lymphocyte-depleted subtype (100\%). Our series also identified lower expression of GLUT1 in the lymphocyte-rich classical cHL subtype (0\%) than in other subtypes.

A GLUT1-specific inhibitor (STF-31) induces apoptosis and sensitizes multiple myeloma cells to conventional chemotherapeutic agents. ${ }^{26} \mathrm{~A}$ recent study found that the GLUT1-specific inhibitor apigenin induces growth retardation and apoptosis through metabolic and oxidative stress caused by the suppression of glucose utilization in lung cancer and adenoid cystic carcinoma. ${ }^{35}$ WZB117 also exerts inhibitory effects on cancer cell growth in breast and lung cancer cell lines. ${ }^{36,37}$ Nivolumab has been tested in patients with advanced $\mathrm{cHL}$, and impressive results were obtained in phase I trial. ${ }^{25}$ Pembrolizumab, an IgG4 monoclonal antibody directed against PD-1, also induced favorable responses in a heavily pretreated patient with $\mathrm{cHL} .^{38}$ Although an objective response was reported in $87 \%$ of patients with advanced cHL treated with nivolumab, only $17 \%$ of patients exhibited complete responses. ${ }^{25}$ Based on the significant correlations of GLUT1 with PD-L1 and PD-L2 in this study and a previous study, ${ }^{24}$ the HIF-2 $\alpha$-GLUT1-PD-L1 pathway may be efficiently inhibited by GLUT1-specific inhibitor and PD-1-specific inhibitor.

In summary, our results suggest that GLUT1 expression is significantly associated with the expression of PD-L1 and PDL2. GLUT1 expression has prognostic significance in advancedstage cHL. Further efforts to understand the mechanisms of the correlations of GLUT1 with PD-L1 and PD-L2 may help the development of effective therapeutic agents for the treatment of $\mathrm{cHL}$.

\section{Conflicts of Interest}

No potential conflict of interest relevant to this article was reported.

\section{Acknowledgments}

This research was supported by a grant from The Korean Society of Pathologists.

\section{REFERENCES}

1. Björkholm M, Axdorph U, Grimfors G, et al. Fixed versus responseadapted MOPP/ABVD chemotherapy in Hodgkin's disease: a prospective randomized trial. Ann Oncol 1995; 6: 895-9.

2. Quddus F, Armitage JO. Salvage therapy for Hodgkin's lymphoma. Cancer J 2009; 15: 161-3.

3. Hasenclever D, Diehl V. A prognostic score for advanced Hodgkin's disease: international prognostic factors project on advanced Hodgkin's disease. N Engl J Med 1998; 339: 1506-14.

4. Barron CC, Bilan PJ, Tsakiridis T, Tsiani E. Facilitative glucose transporters: implications for cancer detection, prognosis and treatment. Metabolism 2016; 65: 124-39.

5. Osugi J, Yamaura T, Muto S, et al. Prognostic impact of the combination of glucose transporter 1 and ATP citrate lyase in node-negative patients with non-small lung cancer. Lung Cancer 2015; 88: 310-8.

6. Pinheiro C, Sousa B, Albergaria A, et al. GLUT1 and CAIX expression profiles in breast cancer correlate with adverse prognostic factors and MCT1 overexpression. Histol Histopathol 2011; 26: 1279-86.

7. Haber RS, Rathan A, Weiser KR, et al. GLUT1 glucose transporter expression in colorectal carcinoma: a marker for poor prognosis. Cancer 1998; 83: 34-40.

8. Kawamura T, Kusakabe T, Sugino T, et al. Expression of glucose transporter-1 in human gastric carcinoma: association with tumor aggressiveness, metastasis, and patient survival. Cancer 2001; 92: 634-41.

9. Hartmann S, Agostinelli C, Diener J, et al. GLUT1 expression patterns in different Hodgkin lymphoma subtypes and progressively transformed germinal centers. BMC Cancer 2012; 12: 586.

10. Shim HK, Lee WW, Park SY, Kim H, Kim SE. Relationship between FDG uptake and expressions of glucose transporter type 1, type 3, and hexokinase-II in Reed-Sternberg cells of Hodgkin lymphoma. Oncol Res 2009; 17: 331-7.

11. Weber J. Immune checkpoint proteins: a new therapeutic paradigm for cancer: preclinical background: CTLA-4 and PD-1 blockade. Semin Oncol 2010; 37: 430-9.

12. Keir ME, Butte MJ, Freeman GJ, Sharpe AH. PD-1 and its ligands 
in tolerance and immunity. Annu Rev Immunol 2008; 26: 677-704.

13. Zeng Z, Shi F, Zhou L, et al. Upregulation of circulating PD-L1/ PD-1 is associated with poor post-cryoablation prognosis in patients with HBV-related hepatocellular carcinoma. PLoS One 2011; 6: e23621.

14. Bellmunt J, Mullane SA, Werner L, et al. Association of PD-L1 expression on tumor-infiltrating mononuclear cells and overall survival in patients with urothelial carcinoma. Ann Oncol 2015; 26: 812-7.

15. Muenst S, Schaerli AR, Gao F, et al. Expression of programmed death ligand 1 (PD-L1) is associated with poor prognosis in human breast cancer. Breast Cancer Res Treat 2014; 146: 15-24.

16. Zhang Y, Wang L, Li Y, et al. Protein expression of programmed death 1 ligand 1 and ligand 2 independently predict poor prognosis in surgically resected lung adenocarcinoma. Onco Targets Ther 2014; 7: 567-73.

17. Droeser RA, Hirt C, Viehl CT, et al. Clinical impact of programmed cell death ligand 1 expression in colorectal cancer. Eur J Cancer 2013; 49: 2233-42.

18. Kumar V, Gabrilovich DI. Hypoxia-inducible factors in regulation of immune responses in tumour microenvironment. Immunology 2014; 143: 512-9.

19. Messai Y, Gad S, Noman MZ, et al. Renal cell carcinoma programmed death-ligand 1, a new direct target of hypoxia-inducible factor-2 alpha, is regulated by von Hippel-Lindau gene mutation status. Eur Urol 2016; 70: 623-32.

20. Noman MZ, Desantis G, Janji B, et al. PD-L1 is a novel direct target of HIF-1alpha, and its blockade under hypoxia enhanced MDSCmediated T cell activation. J Exp Med 2014; 211: 781-90.

21. Barsoum IB, Smallwood CA, Siemens DR, Graham CH. A mechanism of hypoxia-mediated escape from adaptive immunity in cancer cells. Cancer Res 2014; 74: 665-74.

22. Li QQ, Sun YP, Ruan CP, et al. Cellular prion protein promotes glucose uptake through the Fyn-HIF-2alpha-Glut1 pathway to support colorectal cancer cell survival. Cancer Sci 2011; 102: 400-6.

23. Rapisarda A, Uranchimeg B, Scudiero DA, et al. Identification of small molecule inhibitors of hypoxia-inducible factor 1 transcriptional activation pathway. Cancer Res 2002; 62: 4316-24.

24. Ruf M, Moch H, Schraml P. PD-L1 expression is regulated by hypoxia inducible factor in clear cell renal cell carcinoma. Int $\mathrm{J}$ Cancer 2016; 139: 396-403.

25. Ansell SM, Lesokhin AM, Borrello I, et al. PD-1 blockade with nivolumab in relapsed or refractory Hodgkin's lymphoma. N Engl
J Med 2015; 372: 311-9.

26. Matsumoto T, Jimi S, Migita K, Takamatsu Y, Hara S. Inhibition of glucose transporter 1 induces apoptosis and sensitizes multiple myeloma cells to conventional chemotherapeutic agents. Leuk Res 2016; 41: 103-10.

27. Koh YW, Jeon YK, Yoon DH, Suh C, Huh J. Programmed death 1 expression in the peritumoral microenvironment is associated with a poorer prognosis in classical Hodgkin lymphoma. Tumour Biol 2016; 37: 7507-14.

28. Huh J, Cho K, Heo DS, Kim JE, Kim CW. Detection of Epstein-Barr virus in Korean peripheral T-cell lymphoma. Am J Hematol 1999; 60: 205-14.

29. Pommier Y, Leo E, Zhang H, Marchand C. DNA topoisomerases and their poisoning by anticancer and antibacterial drugs. Chem Biol 2010; 17: 421-33.

30. Jordan MA, Wilson L. Microtubules as a target for anticancer drugs. Nat Rev Cancer 2004; 4: 253-65.

31. Koch A, Lang SA, Wild PJ, et al. Glucose transporter isoform 1 expression enhances metastasis of malignant melanoma cells. Oncotarget 2015; 6: 32748-60.

32. Yan S, Wang Y, Chen M, Li G, Fan J. Deregulated SLC2A1 promotes tumor cell proliferation and metastasis in gastric cancer. Int J Mol Sci 2015; 16: 16144-57.

33. Uddin S, Hussain AR, Ahmed M, et al. Inhibition of c-MET is a potential therapeutic strategy for treatment of diffuse large B-cell lymphoma. Lab Invest 2010; 90: 1346-56.

34. Dinand V, Malik A, Unni R, Arya LS, Pandey RM, Dawar R. Proliferative index and CD15 expression in pediatric classical Hodgkin lymphoma. Pediatr Blood Cancer 2008; 50: 280-3.

35. Lee YM, Lee G, Oh TI, et al. Inhibition of glutamine utilization sensitizes lung cancer cells to apigenin-induced apoptosis resulting from metabolic and oxidative stress. Int J Oncol 2016; 48: 399-408.

36. Liu Y, Cao Y, Zhang W, et al. A small-molecule inhibitor of glucose transporter 1 downregulates glycolysis, induces cell-cycle arrest, and inhibits cancer cell growth in vitro and in vivo. Mol Cancer Ther 2012; 11: 1672-82.

37. Zhao F, Ming J, Zhou Y, Fan L. Inhibition of Glut1 by WZB117 sensitizes radioresistant breast cancer cells to irradiation. Cancer Chemother Pharmacol 2016; 77: 963-72.

38. Armand P, Shipp MA, Ribrag V, et al. Programmed death-1 blockade with pembrolizumab in patients with classical Hodgkin lymphoma after brentuximab vedotin failure. J Clin Oncol 2016 Jun 27 [Epub]. https://doi.org/10.1200/JCO.2016.67.3467. 